\title{
Análisis de necesidades e intereses recreativos de las personas adultas mayores del albergue San José Obrero, del cantón de Siquirres: propuesta recreativa
}

\author{
Analysis of Recreational Necessities and Interests of \\ Senior Citizens from "Albergue San José Obrero" \\ in Siquirres: Recreational Proposal
}

\author{
Grettel Romero Morales \\ Liceo de Pocora \\ Limón, Costa Rica \\ grerm@hotmail.com
}

Recibido 03-06-2010 • Aceptado 14-10-2010 • Corregido 15-11-2010

\begin{abstract}
Resumen: Este artículo comprende el análisis de las necesidades y los intereses recreativos de las personas adultas mayores del "Albergue San José Obrero" del cantón de Siquirres, y una propuesta recreativa que se ajusta a sus requerimientos. El enfoque utilizado en esta investigación fue cualitativo de investigaciónacción y se utilizó la entrevista cualitativa, la discusión de grupo focal, la lista de cotejo y el diario de campo como instrumentos de medición. La información acerca de las necesidades e intereses recreativos se obtuvo por medio de un diagnóstico institucional, la información de la condición general de las personas adultas mayores y las opiniones de las personas adultas mayores acerca de sus experiencias y preferencias recreativas. La información obtenida se analizó mediante las técnicas de resumen y de sistematización. El estudio mostró la necesidad en esta población de mantener su condición física y cognoscitiva, su vinculación con la sociedad, el sentido de pertenencia al grupo y el sentido de utilidad y autosuficiencia; además del interés por participar en manualidades, paseos, actividades de aprendizaje, actividad física, siembra, pesca, cuentos, bingo y actividades culinarias. Con las conclusiones derivadas del diagnóstico se elaboró una propuesta recreativa que comprende la descripción detallada de 12 actividades recreativas para ser implementadas en la población investigada.
\end{abstract}

\section{Introducción}

Las condiciones económicas y los avances tecnológicos entre otros factores contribuyen a un acelerado envejecimiento de la población, que a su vez exige la necesidad de generar oportunidades que permita a la sociedad adaptarse a esta condición. La educación, como medio transformador en las comunidades, posee un gran desafío en la proyección, generación y organización de las mejores condiciones y oportunidades para que esta población tenga la posibilidad de concretar sus potencialidades, de crecer, de crear, de proyectar y proyectarse para su propio bienestar y el de las comunidades (Tamer, 1999).

Sin embargo, en la actualidad, después de los 65 años de edad se retira de su trabajo a la mayoría de personas, se le hace dependiente de algún familiar, se le confina a esperar su defunción en su casa o 
Las actividades recreativas propuestas comprenden inducción a la recreación, actividades culinarias, manualidades, juegos de mesa, paseo, siembra, actividades físicas e intelectuales.

Palabras clave: Recreación, persona adulta mayor, propuesta recreativa, necesidades recreativas, intereses recreativos.

\begin{abstract}
This article includes the analysis of the recreational necessities and interests of senior citizens at "Albergue San José Obrero" in Siquirres, and a recreational proposal that meets their requirements. The approach used in this research was an investigative-action qualitative research, thus qualitative interviews, focus group discussions, checklists and field diaries were used as measuring instruments. Information about recreational necessities and interests were obtained through an institutional analysis, information about the general condition of the elderly and the opinions from older people about their recreational experiences and preferences. The data was analyzed using the summary and systematization techniques. The study showed the necessities in this population to maintain their physical and cognitive status, their relationship with society, their sense of belonging, a sense of usefulness and self-sufficiency; plus interest to participate in crafts, outings, learning activities, physical activities, planting, fishing, storytelling, bingo and cooking activities. Based on the conclusions of the diagnosis, a recreational proposal was elaborated with the description of twelve recreational activities to be implemented in the study population. The proposed activities include: induction to recreation, cooking activities, crafts, games, walks, planting, physical and intellectual activities.
\end{abstract}

Key words: Recreation, senior citizens, recreational activities, recreational necessities, recreational interests. en algún albergue sin mayores aspiraciones o regocijo por la vida (Ballestero, 1999).

Estas condiciones propician en este grupo etario un incremento de enfermedades físicas y emocionales en detrimento de su calidad de vida, por lo que la implementación de programas recreativos es una alternativa que podría contribuir a mejorar su condición y su desenvolvimiento pleno como ser humano en la sociedad (Ballestero, 1999; Marín y García, 2004).

En este sentido, algunos escritores, como Rico (1999), Salazar (2007), Stumbo (2000) y Thang (2005), consideran a los programas recreativos como fuente de beneficios ilimitados que contribuyen a mantener y mejorar la calidad de vida mediante la interacción social y la realización personal de la población en general. Además, coinciden en que los beneficios atribuidos a la práctica de actividades recreativas son innumerables y únicos para cada persona, por lo que la participación frecuente en este tipo de actividades favorece el crecimiento personal, social, psicológico, emocional y espiritual del individuo.

\section{Justificación}

Al considerar las condiciones de vida de la población adulta mayor y los beneficios que podrían adquirir con la participación en programas recreativos, se elaboró un estudio de las necesidades e intereses recreativos de las personas adultas mayores del Albergue San José Obrero del cantón de Siquirres, para desarrollar una propuesta recreativa que se ajustara a sus necesidades.

Esta propuesta pretende que la participación en las actividades recreativas les permita mejorar sus relaciones interpersonales, aumentar su confianza, fortalecer su autoestima, y ayude a mantener sus capacidades cognoscitivas, físicas y emocionales, además de mejorar su calidad de vida (Butts y Chana, 2007). 
Asimismo, será una oportunidad para que la institución pueda cumplir con la ley 7935, Ley Integral para la Persona Adulta Mayor, que en el Artículo 3 apunta: "Toda persona adulta mayor tendrá derecho a una mejor calidad de vida mediante la creación y ejecución de programas que promuevan la participación en actividades recreativas, culturales y deportivas, promovidas por las organizaciones, las asociaciones, las municipalidades y el Estado" (Consejo Nacional de la Persona Adulta Mayor, CONAPAM, 1999).

De igual forma, el programa recreativo propuesto podría contribuir con la calidad de vida de las personas adultas mayores del hogar y podría servir de modelo a otras instituciones con población en condiciones similares. A la vez, el desarrollo de esta propuesta permitirá al Comité Cantonal de Deportes y Recreación de Siquirres crear un precedente recreativo con este tipo de población.

Los objetivos de este artículo son:

1. Determinar las necesidades y los intereses recreativos de las personas adultas mayores del Albergue San José Obrero.

2. Elaborar una propuesta de un programa recreativo que contribuya a mejorar la calidad de vida de la persona adulta mayor del Albergue San José Obrero.

La orientación teórica de este artículo considera a la población adulta mayor y sus condiciones de vida, las actividades recreativas y los beneficios para las personas adultas mayores además de las características de la programación de actividades recreativas.

\section{Población adulta mayor y sus condiciones de vida}

En América Latina, hay 40 millones de personas adultas mayores. Esta población representa el 8\% de la población total y, según predicciones con respecto al índice de natalidad y mortalidad, se espera que, para el año 2025, alcance un 14\% de la población total (Ocampo, 2004). En Costa Rica, según el Instituto Nacional de Estadística y Censos (INEC), en el año 2000 la población adulta mayor representó un 7,9\% (300.000 personas aproximadamente) de la población total y se predice que, para el 2025, alcance un $14,4 \%$ de la población total, tal y como se muestra en la siguiente tabla estadística (Costa Rica. INEC, 2008).

Tabla 1

Distribución por edades de la población costarricense 1950-2025

\begin{tabular}{llcc}
\hline Año & $0-14$ & $15-59$ & 60 y más \\
\hline 1950 & $43,3 \%$ & $51,0 \%$ & $5,7 \%$ \\
1970 & $46,1 \%$ & $49,0 \%$ & $5,0 \%$ \\
1980 & $38,8 \%$ & $55,6 \%$ & $5,6 \%$ \\
1995 & $34,5 \%$ & $58,6 \%$ & $6,9 \%$ \\
2000 & $32,0 \%$ & $60,0 \%$ & $7,9 \%$ \\
2025 & $24,3 \%$ & $61,4 \%$ & $14,4 \%$ \\
\hline
\end{tabular}

Fuente: Costa Rica. Ministerio de Planificación Nacional y Política Económica (2008) y Costa Rica. Instituto Nacional de Estadística y Censos (2008).

La proyección expuesta en la anterior tabla estadística muestra un creciente envejecimiento poblacional importante de considerar si se toma en cuenta las condiciones biológicas y psicológicas que se presentan en la adultez mayor.

Estas condiciones presentes en la adultez mayor son consideradas producto del proceso de envejecimiento que se manifiesta paulatinamente durante el transcurso de la vida de las personas y, en la etapa de la adultez mayor, se evidencia por medio de cambios biológicos (Ballestero, 1999; Escobar, 2003; Restrepo, Morales, Ramírez, López y Varela, 2006) como: 
- Disminución de la velocidad y longitud del paso.

- Aumento en la aparición de enfermedades crónicas.

- Reducción de la masa y la fuerza musculares.

- Modificaciones en el sistema respiratorio.

- Disminución de la capacidad auditiva y visual, entre otras.

Al tiempo que se presentan las condiciones biológicas en la adultez mayor, surgen características emocionales como baja autoestima, depresión, tristeza crónica, dependencia y, en algunos casos, detrimento del sentido de la vida (Ballestero, 1999; Escobar, 2003).

Estas condiciones biológicas y psicológicas que genera la adultez mayor demandan estimular la recreación como un estilo de vida en la población y crear los servicios recreativos necesarios que garanticen su calidad de vida.

\section{Actividades recreativas y beneficios de la recreación para la persona adul- ta mayor}

La recreación como estilo de vida es fundamental para el bienestar de las personas (Malmberg, Miilumpalo, Pasanen, Vouri y Oja, 2006). A menudo, proporciona un ambiente en el que la persona puede experimentar interacción social, felicidad, desafíos, mejora de la habilidad, sentido de logro y libertad para elegir (Rico, 1999; Mora, 2002). Los beneficios atribuidos a la recreación son considerados infinitos y únicos para cada persona (Stumbo, 2000).

Las personas adultas mayores se encuentran facultadas para participar en diversas actividades recreativas. Los juegos, las manualidades, los paseos, la actividad física, la música y la danza son ejemplos de las muchas formas en las que pueden utilizar su tiempo libre, el cual es considerado como el periodo que no se utiliza para el trabajo, aseo personal, alimentación o estudio (Garita, Godínez, López, Navarro y Sibaja, 2004; Salazar, 2007).

Una constante participación en actividades recreativas les permite a las personas adultas mayores mantener la habilidad de vivir independientemente, reducir el riesgo de sufrir fracturas, disminuir los síntomas de depresión y a vivir de manera plena (Escobar, 2003).

\section{Actividades recreativas}

Estas actividades son las que se realizan durante el tiempo libre; contribuyen al desarrollo personal, espiritual y social del individuo y son fundamentales para el ser humano por sus aportes al desarrollo integral de las personas (Garita et al, 2004; Salazar, 2007). Las actividades recreativas, según Salazar (2007), pueden clasificarse en diferentes categorías:

a. Deportes, juegos y actividades físicas.

b. Actividades artísticas

c. Recreación social

d. Actividades al aire libre y relacionadas con la naturaleza

e. Actividades cognitivas

f. Actividades de enriquecimiento y actualización personal

g. Pasatiempos

h. Turismo

Cuando se implementan actividades recreativas, es de suma importancia reconocer la intencionalidad o el objetivo que se desea alcanzar. Este tipo de actividades debe ser trascendental, cumplir con el objetivo de divertir y, a la vez, producir un cambio en las actitudes, el conocimiento y las conductas de la población participante (Stumbo, 2000). Por otra parte, Los Santos (2002) recomienda, además, tomar en cuenta los siguientes criterios en la formulación de actividades recreativas dirigidas a personas adultas mayores: 
a. Prever la viabilidad de las actividades de acuerdo con las destrezas de la población.

b. Tener en cuenta la falta de cultura recreativa.

c. Procurar que los objetivos de las actividades respondan a las necesidades de la población participante.

d. Tratar en forma integral los aspectos biológicos, sociológicos y psicológicos.

e. Desarrollar una adecuada motivación para conseguir una buena dinámica de grupo.

f. Permitir a la población participante la libertad de desarrollar sus propias estrategias en la realización de las actividades.

g. Mantener coherencia, continuidad y progresión en el programa.

h. Evaluar constantemente con el fin de reivindicar la continuidad del programa.

\section{Beneficios de la recreación}

Los beneficios que se obtienen de la recreación permiten a la persona adulta mayor mejorar la calidad de vida, gozar de salud física y mental y un adecuado desarrollo social, entre otros (Cárdenas y Osorio, 2006). Estos beneficios se pueden clasificar, según Rico (1999) y Salazar (2007), en personales y sociales.

\section{Beneficios personales}

De acuerdo con Rico (1999), los beneficios contribuyen al buen estado físico, sociopsicológico, intelectual y espiritual de los individuos. De estos pueden citarse:

1. Mejor la salud mental y el mantenimiento de esta, lo cual proporciona sentido general de bienestar, manejo adecuado del estrés, reducción de la depresión, la ansiedad, el enojo, entre otros.
2. Desarrollo y crecimiento personal por medio de la generación de autoconfianza, independencia, clarificación de valores, mejoramiento académicocognitivo, autonomía, independencia, sentido de control sobre la propia vida, humildad, liderazgo, aumento de la capacidad estética, aumento de la creatividad, crecimiento espiritual, adaptabilidad, habilidad para resolución de problemas, comprensión ambiental, tolerancia, aceptación de las propias responsabilidades, sentido de libertad, euforia, estimulación, sentido de aventura, desafíos, nostalgia, expresión creativa, apreciación estética, apreciación natural, espiritualidad y cambios positivos de las emociones.

\section{Beneficios sociales}

Los beneficios sociales son aquellos que la sociedad experimenta por medio del fortalecimiento de redes de activismo comunitario y el compromiso que adquieren las personas con su comunidad (Rico, 1999). Según Salazar (2007), son catalogados como los beneficios que obtienen la familia, los grupos sociales, la economía y el ambiente.

Por lo tanto, la práctica de actividades recreativas se puede considerar como una excelente alternativa que les permite a las personas adultas mayores desenvolverse personal y socialmente de forma óptima, al mismo tiempo que contribuye al desarrollo integral de la familia, al crecimiento económico de la nación y a la protección del ambiente.

Por otra parte, la realización de actividades recreativas que conlleve obtener un beneficio asociado requiere una planificación previa para alcanzar objetivos concretos. En el planeamiento de un programa recreativo se puede considerar la intervención de la población meta o la participación de una persona experta en Recreación. 


\section{Planeamiento del programa recreativo}

El planeamiento en la recreación es considerado como uno de los procedimientos necesarios para realizar las actividades recreativas y alcanzar sus objetivos (Jordan, DeGraaf y DeGraaf, 2005; Salazar, 2008; Sport and Recreation Queensland, 2008).

La variación del planeamiento de las actividades recreativas depende del grado de participación de la población meta en la elaboración del programa recreativo (Jordan, DeGraaf y DeGraaf, 2005). El planeamiento que se realiza sin la intervención de las personas participantes suele ser propuesto por la persona profesional en recreación con la intención de resolver algún problema. En contraposición, se encuentra el planeamiento que utiliza la intervención de la persona participante, el cual se ajusta a las necesidades particulares de una población previamente definida y diagnosticada (Salazar, 2008).

El desarrollo del planeamiento, partiendo del modelo en el que se utiliza la intervención del participante, suele realizarse según los siguientes pasos (Salazar, 2008):

1. Identificar y realizar el diagnóstico de necesidades

2. Redactar los objetivos

3. Programar

4. Poner en práctica el programa

5. Evaluar el programa

6. Tomar decisiones acerca del programa

\section{Metodología}

\section{Tipo de investigación}

La investigación se orientó por un enfoque cualitativo. Según Hernández, Fernándezy Baptista(2006), lainvestigación cualitativa establece conceptos esenciales para iniciar la investigación en lugar de variables exactas; se aplica a un número reducido de casos; los significados se extraen de los datos; no se fundamenta en la estadística y está sujeta a las circunstancias de cada ambiente.

El método por utilizar en este proyecto es investigación-acción, el cual se centra en aportar información que guíe la toma de decisiones y los procesos de cambio para su mejora (Sandín, 2003).

El proceso de investigación-acción, según Sandín (2003), es concebido de diversas formas; sin embargo, la conceptualización más generalizada es el modelo de "espiral de ciclo", que consta de cuatro etapas:

1. Identificación del problema de investigación

2. Elaboración de un plan de acción

3. Implementación del plan y evaluación de resultados

4. Interpretación de resultados y replanificación

La selección de este enfoque de investigación obedece, principalmente, a las condiciones de la población de estudio. La escasa o inexistente experiencia en actividades recreativas les impedía, en este caso, dar un criterio fundamentado sobre sus intereses y necesidades recreativas, por lo que requerían de la participación en diferentes escenarios recreativos para opinar acerca de su experiencia.

Parte de este estudio consistió en realizar un diagnóstico, el cual brindó información acerca de las necesidades y los intereses recreativos de las personas adultas mayores para la elaboración de la propuesta del programa recreativo.

\section{Fases de la investigación}

Al seguir el modelo del proceso de investigación-acción, el desarrollo de la investigación comprendió diferentes actividades en cada etapa, como se muestra en el cuadro siguiente: 
Tabla 2

Fases de la investigación

\begin{tabular}{|c|c|}
\hline Etapa & Actividades \\
\hline $\begin{array}{l}\text { 1. Identificación del problema de } \\
\text { investigación }\end{array}$ & $\begin{array}{l}\text { En esta etapa inicial se realizó: } \\
\text { a. La entrevista con la administradora acerca de la } \\
\text { información institucional. } \\
\text { b. La entrevista con la enfermera para obtener la información } \\
\text { general de las personas adultas mayores del Albergue. } \\
\text { c. La entrevista con las personas adultas mayores quienes } \\
\text { participaron en la actividad, con la finalidad de conocer } \\
\text { sus experiencias e intereses recreativos. }\end{array}$ \\
\hline 2. Elaboración de un plan de acción & $\begin{array}{l}\text { Se analizó la información obtenida en la etapa anterior (entre- } \\
\text { vistas a la administradora, a la enfermera y a las personas } \\
\text { adultas mayores) para la selección de las seis actividades } \\
\text { recreativas de diagnóstico y su respectiva programación. }\end{array}$ \\
\hline $\begin{array}{l}\text { 3. Implementación del plan y } \\
\text { evaluación de resultados }\end{array}$ & $\begin{array}{l}\text { Se puso en práctica el programa de las actividades recreativas } \\
\text { de diagnóstico y se evaluó por medio de la discusión del grupo } \\
\text { focal, la lista de cotejo y el diario de campo. }\end{array}$ \\
\hline $\begin{array}{l}\text { 4. Interpretación de resultados y } \\
\text { replanificación }\end{array}$ & $\begin{array}{l}\text { Se analizaron los resultados y se elaboró la propuesta del } \\
\text { programa recreativo. }\end{array}$ \\
\hline
\end{tabular}

Fuente: elaboración propia.

\section{Rol de la persona investigadora}

La persona investigadora participó en el proceso en forma activa; aplicó los instrumentos de evaluación para la recolección de información y, además, seleccionó y coordinó las actividades recreativas de diagnóstico.

\section{Participantes y su selección}

El estudio se realizó en el Albergue de Ancianos San José Obrero, de Siquirres. Para tal efecto se consideró la participación de las siguientes personas:

a. Diez personas adultas mayores que desearan participar y que tuvieran la autorización de la enfermera de la Institución. La selección de la población adulta mayor participante obedeció, principalmente, a las recomendaciones que realizó la enfermeradurantela entrevista acerca de las condiciones de salud, tanto físicas como cognoscitivas mínimas de lucidez que les permitieran a las personas responder las preguntas de las entrevistas por realizar.

La población residente en el hogar presenta condiciones de presión alta (HTA), colesterol, enfermedad pulmonar crónica (EPOC), vértigo, diabetes, artrosis, bajo peso, intestino irritable, gastritis, dolor de cabeza, incapacidad visual, esquizofrenia, entre otras.

La escolaridad de la población participante es reducida; ninguna persona terminó el $6^{\circ}$ grado de la escuela. Algunas personas no saben leer ni escribir o se les dificulta por la disminución de la capacidad visual relacionada a enfermedades como la diabetes.

b. La enfermera de la Institución.

c. La administradora de la Institución. 


\section{Técnicas o instrumentos empleados}

Los instrumentos de recolección de información que se utilizaron en la investigación fueron:

1. Entrevista cualitativa. En el estudio se realizaron cuatro entrevistas:

a. Entrevista a la administradora del Albergue para conocer aspectos generales de la Institución, como antecedentes, instalaciones, recurso humano, financiamiento, reglamentos, así como otros aspectos importantes que surgieron durante la entrevista.

b. Entrevista a la enfermera de la Institución para recopilar información general acerca de las personas adultas mayores residentes del Albergue como nombre completo, edad, fecha de nacimiento, sexo, enfermedad que padece, tratamientos, capacidad física, escolaridad, actividades que realiza durante su tiempo libre y otros aspectos que la persona entrevistada consideró importante.

c. Entrevista a las personas adultas mayores del Albergue quienes participaron en el estudio con el objetivo de conocer sus necesidades e intereses recreativos por medio de información del lugar donde vivía cuando era niño, de que manera se entretenía en la niñez, juventud y adultez, además de las actividades en las que le interesa y no le interesa participar en la actualidad.

d. La entrevista que se pretendió realizar a los familiares de las personas participantes en el estudio consistía en conocer acerca de las actividades en las que solían participar los sujetos de estudio.
2. Lista de cotejo de las actitudes observadas por la persona evaluadora en las personas participantes durante las sesiones recreativas. Instrumento que presenta un encabezado, objetivos, indicaciones, rasgos observables y criterios de valoración (participó en la actividad, mantuvo la atención, siguió indicaciones, concluyó la actividad, brindó apoyo y mostró satisfacción).

3. Discusión de grupo focal. En esta investigación, la discusión de grupo focal se realizó al finalizar cada sesión recreativa con el propósito de conocer la opinión de las personas adultas mayores participantes acerca de la experiencia vivida. Se consideró la motivación para participar en la actividad, el agrado experimentado, el reconocimiento del beneficio obtenido, las sugerencias a las actividades, la participación en futuras actividades recreativas similares a las realizadas.

4. Diario de campo. En el diario de campo se registraron los detalles sucedidos durante la realización de las actividades recreativas de diagnóstico en función de las necesidades y los intereses recreativos de las personas participantes. La información del diario de campo se recopiló después de realizar cada sesión recreativa. La estructura del diario de campo comprendió nombre de la actividad, fecha, crónica de la actividad, comentarios realizados por las personas participantes y observaciones.

5. Grabaciones de vídeo y audio. Se grabaron las entrevistas y las actividades recreativas de diagnóstico para apoyar la realización de resúmenes y análisis de la información. Las producciones documentales no fueron utilizadas con fines lucrativos, ni para cualquier otra finalidad que no se haya especificado en esta investigación; las grabaciones de audio y vídeo se destruyeron después de realizado el estudio. 


\section{Procedimientos de recolección de la información}

El diagnóstico consistió en:

a. Realización de entrevista a la administradora para conocer aspectos generales de la Institución.

b. Ejecución de entrevista a la enfermera de la Institución para conocer información general de las personas adultas mayores del Albergue.

c. Entrevistas a las personas adultas mayores del Albergue para conocer sus experiencias e intereses recreativos.

d. Se consideró entrevistar a los familiares de las personas quienes participaron en el estudio para conocer acerca de las actividades en las que solía participar la población de estudio antes de llegar a la Institución. Estas entrevistas no se realizaron.

e. Ejecución de seis sesiones recreativas de diagnóstico que permitieron evaluar los intereses y las necesidades de las personas participantes por medio de la discusión de grupo focal, la lista de cotejo y el diario de campo.

\section{Procedimientos de análisis de la información}

El análisis de la información de las entrevistas se realizó por medio del resumen y la discusión de grupo focal, la lista de cotejo y el diario de campo mediante la sistematización. La sistematización permite la interpretación crítica de una o varias experiencias que, a partir de la reconstrucción, descubre la lógica del proceso vivido y los factores que han intervenido en el proceso (Jara, 1998).

Entrevista a la administradora de la Institución

De la entrevista que se le realizó a la administradora, se analizaron los siguientes aspectos:
1. Áreas posibles de desarrollo de actividades recreativas.

2. Salidas de emergencia y zonas de seguridad.

3. Personas con las que se podía contar como apoyo en las actividades.

4. Financiamiento del programa.

5. Horarios de actividades del hogar.

\section{Entrevista a la enfermera de la Institución}

La entrevista a la enfermera se analizó los siguientes aspectos:

1. Posibles personas participantes en el estudio.

2. Tratamientos recibidos y cuidados que requerían.

3. Condiciones físicas, capacidades cognoscitivas y sociales.

Entrevista a las personas adultas mayores quienes participaron en las actividades recreativas de diagnóstico

Esta entrevista se resumió y, de la información obtenida, se analizaron los siguientes aspectos:

1. Actividades recreativas en las que habían participado las personas entrevistadas.

2. Actividades recreativas en las que les gustaría participar.

3. Actividades recreativas en las que no les gustaría participar.

Esta información se utilizó para la programación de seis sesiones de actividades recreativas.

Discusión de grupo focal, diario de campo y lista de cotejo

El objetivo de la sistematización fue reconstruir la experiencia vivida durante el proceso de intervención con el fin de 
identificar las necesidades y los intereses recreativos de la población en estudio.

De la discusión de grupo focal se analizaron los siguientes aspectos:

1. La motivación para participar en la actividad

2. La satisfacción experimentada por las personas participantes

3. La utilidad percibida por las personas participantes de la actividad (beneficios obtenidos)

4. Las sugerencias realizadas a la actividad para su mejora

5. El interés por participar en ese tipo de actividades

\section{Triangulación de la información}

La triangulación para validar la información se llevó a cabo comparando los resultados de las entrevistas realizadas a las personas adultas mayores y los resultados obtenidos de las actividades recreativas de diagnóstico. La triangulación de la información contribuyó a ajustar fielmente a la realidad de la población la información obtenida acerca de sus necesidades y sus intereses recreativos.

\section{Resultados}

La información obtenida en la investigación expone aspectos generales de la población de estudio, sus condiciones físicas y cognoscitivas además de sus necesidades e intereses recreativos.

\section{Resultado del análisis de la entrevista a la persona administradora de la Institución}

El Albergue San José Obrero está ubicado en Barrio Brooklyn, del cantón de Siquirres; posee una población de 22 personas adultas mayores residentes, con edades entre los 68 y 98 años.
La Institución cuenta con amplias zonas verdes, un salón para reuniones, pasillos espaciosos y el comedor, los cuales podrían utilizarse para la realización de actividades recreativas.

El albergue posee dos zonas internas de seguridad y señalización de rutas de evacuación. Además, cuenta con seis extintores contra incendio, distribuidos en lugares accesibles de las instalaciones.

Las personas que podrían brindar apoyo en la realización de actividades son la encargada del bienestar de las personas adultas mayores del albergue, junto con la persona asesora recreativa del Comité Cantonal del Deportes y Recreación de Siquirres.

Además, durante el año la Institución recibe servicio de voluntariado de estudiantes de colegios y de universidades quienes realizan sus trabajos comunitarios en la Institución.

La Institución no cuenta con un rubro destinado para el financiamiento de un programa recreativo institucional, por lo que se requiere la colaboración de organismos como el Consejo Nacional de la Persona Adulta Mayor (2008), la Asociación Gerontológica Costarricense (AGECO, 2008), la Municipalidad de Siquirres, el Comité de Deportes y Recreación de Siquirres y la empresa privada.

\section{Resultados del análisis de las entrevistas a las personas adultas mayores participantes}

De acuerdo con las entrevistas realizadas a las personas adultas mayores, las señoras recuerdan que, en su infancia, les agradaba bañarse en el río, jugar con muñecas y hacerles la ropa; cuando eran jóvenes les agradaba bailar, cantar y participar en las serenatas.

"Íbamos a bañarnos al río cuando estábamos en la escuela" (María, 93 años). 
"A veces los novios llevaban serenatas, bonito eran las serenatas a la media noche" (María, 93 años).

Los señores entrevistados recuerdan que les agradaba el baile, la pesca, nadar en los ríos, ir a serenatas, sembrar, jugar fútbol, ir de cacería, tocar guitarra, cantar y asistir a los turnos en las comunidades.

"Bailé hasta lo último que podía haber, a mí no me decían el nombre, solo me decían movida. ...no llegó movida hay que buscarlo" (Mario, 72 años).

"Yo iba a cacería sí, iba a cazar tepezcuintles" (Carlos, 68 años).

Las personas entrevistadas mencionaron que, en la actualidad, durante su tiempo libre escuchan música, conversan, observan la televisión y duermen.

Comentan que, además, esporádicamente participan en actividades manuales que algunos grupos de estudiantes ofrecen en la Institución. La mayoría de personas entrevistadas reconoció la necesidad de realizar alguna actividad durante su tiempo libre y expresaba gran disposición para participar en el proyecto, independientemente de las actividades que se realizaran. "Claro, para tener algo que hacer" (Mario, 72 años).

Las señoras entrevistadas explicaron que querían realizar pinturas, coser, participar en serenatas, salir de paseo, hacer actividad física y bailar. Los señores dijeron saber cocinar y que les agradaría hacerlo, además de pasear, pescar, pintar y sembrar.

"Nosotros cocinamos, sí yo cocino, yo allá donde teníamos la finca, yo cocino" (Carlos, 68 años).

La mayor parte de las personas entrevistadas expresó agrado por las actividades recreativas, no obstante, tres de ellas reconocieron no gustarle una actividad específica. Una persona indicó no agradarle cocinar y preferir las actividades del campo; otra expresó desinterés por la pesca y uno más por las manualidades.

\section{Resultados de las seis sesiones recreativas}

Metodología de las sesiones recreativas de diagnóstico

La selección de las actividades recreativas de diagnóstico obedeció, principalmente, a las acciones que realizaron las personas entrevistadas durante su juventud e infancia para ocupar su tiempo libre y los recursos disponibles de la zona; además, se tomaron en consideración las recomendaciones realizadas por Los Santos (2002) acerca de tener en cuenta la falta de cultura recreativa, prever la viabilidad de las actividades de acuerdo con las destrezas y las necesidades de la población, así como desarrollar una adecuada motivación para conseguir una buena dinámica de grupo.

La primera sesión recreativa se denominó "Construyo con mis manos". Las actividades recreativas de diagnóstico de esta sesión comprendían armar y decorar un cajón de madera o elaborar ropa para muñeca, con la finalidad de estimular la motora fina y la imaginación. La sesión se realizó durante tres horas y se contó con la asistencia de ocho personas participantes.

La segunda sesión se denominó "Acondiciono mi cuerpo". Las actividades recreativas de esta sesión consistían en la realización de actividades físicas de desplazamiento (búsqueda del tesoro con banderines de colores instalados alrededor de las instalaciones, los cuales contenían una pieza de un rompecabezas que debían armar) y movilización de extremidades superiores e inferiores (voleibol). La sesión se desarrolló durante dos horas y se contó con la asistencia de ocho personas participantes. 
La tercera sesión, llamada "El paseo", se realizó con el objetivo de brindarles a las personas participantes un acercamiento con la sociedad y un cambio de entorno. En esta sesión recreativa se trasladó a las personas participantes a un centro recreativo en donde se les ofrecieron actividades físicas, artísticas, visita a un mariposario, además del tiempo para compartir la merienda y el almuerzo. El paseo se realizó durante cuatro horas y se contó con la asistencia de las 10 personas participantes del estudio.

La cuarta sesión: "Preparo el pan para el café de la tarde", procuró fortalecer la autoestima, las destrezas motoras y el sentido de productividad por medio de la realización de repostería con la que se podría tomar el café de la tarde. Se contó con la asistencia de seis personas participantes, con una duración de dos horas.

La quinta sesión se realizó con el objetivo de fortalecer el sentido de utilidad de las personas participantes y de responsabilidad con el medio ambiente, se brindó una charla motivacional de la importancia de los árboles, así como de la siembra y el cuidado permanente de un arbusto. La sesión se denominó "Contribuyo con el medio ambiente", se contó con la asistencia de nueve participantes y se desarrollo durante dos horas.

La sexta sesión se denominó "Noche artística”. Esta actividad comprendió la exposición de talentos por parte de las personas adultas mayores de la Institución. Se contó con la animación de un grupo de guitarristas, la exposición de malabares con fuego, cuentachistes, comida y obsequios. Esta actividad se realizó con el objetivo de fortalecer el sentido de utilidad y la convivencia con personas de la comunidad. A la actividad se hicieron presentes los diez participantes y se llevó a cabo durante tres horas.

\section{Resultados}

La cocina y el paseo fueron las actividades en las que las personas participantes mostraron mayor concentración, energía, interés y satisfacción. Para las personas adultas mayores del estudio, sentirse útiles e interactuar con otras personas es de suma importancia.

Las necesidades recreativas de esta población son eminentes debido a la carencia en la Institución de actividades que les permita a las personas adultas mayores mantener su condición física y cognoscitiva, su vinculación con la sociedad, el sentido de pertenencia al grupo, el sentido de utilidad y la autosuficiencia.

Los intereses recreativos mostrados por las personas adultas mayores comprendieron las manualidades, los paseos, el aprendizaje, la interacción con la sociedad, la música, el baile, la actividad física, la siembra, la pesca, los cuentos, el rompecabezas, el bingo y la cocina.

En las actividades recreativas se mantuvo el desinterés de una de las personas participantes en realizar manualidades. La oposición de participar en actividades de pesca y cocina de dos personas participantes, según las entrevistas, desapareció en el momento de la realización de la actividad.

Además, se mostró resistencia por parte de una persona participante de ensuciarse las manos en la actividad de siembra, rechazo que no se mencionó en la entrevista.

\section{Consideraciones finales}

a. Los beneficios infinitos que se obtienen al participar en actividades recreativas podrían convertirse en una excelente alternativa que contribuya a incrementar la calidad de vida de las personas adultas mayores de la institución. Según Márquez y Meléndez (2002), la recreación conforma un conjunto de actividades que las personas adultas mayores requieren de manera sistemática e inmediata porque contribuye, de manera determinante, en mantener la calidad de vida. 
b. Es necesario implementar programas recreativos autónomos en cada centro de atención para la persona adulta mayor, debido a la futura creciente demanda de estos servicios. Según las proyecciones realizadas por el Instituto Nacional de Estadística y Censo (Costa Rica. INEC, 2008) y el Centro Centroamericano de Población (CCP) (Costa Rica, CCP, 2009), la estructura de edades para el año 2050 en Costa Rica mostrará un cambio importante, pasará de un $6,4 \%$ de la población total, en el 2009, a representar entre un $18 \%$ y un $23 \%$ de la población total en el 2050 (Costa Rica, CCP, 2009).

c. La población adulta mayor que reside en hogares de ancianos dispone de gran cantidad de tiempo libre que, bien canalizado, puede ser aprovechado para un adecuado desarrollo personal y social. De acuerdo con lo expuesto por Ferreyra, Choque y Velazco (2005), se ha llegado a descubrir dentro de las instituciones gerontológicas que algunas personas mayores permanecen pasivas gran parte del tiempo, sin la motivación necesaria para accionar de forma positiva, y que muchas personas adultas mayores activas demuestran gran capacidad creativa. La promoción de la salud y la prevención de enfermedades en la población adulta mayor persiguen que a este grupo etario se le proporcione un mejor control de la salud y, al mismo tiempo, que mejore la calidad de vida mediante el empleo adecuado del tiempo libre en actividades físicas, en integración social, entre otras (Figueredo, Sotolongo, Arcias y Díaz, 2003).

d. La adecuada motivación y la justificación de las actividades por realizar aumentan el interés de las personas participantes; convencerlas de sus capacidades para alcanzar metas, a pesar de su condición física, influye positivamente en el éxito del programa, indistintamente de la actividad por ejecutar.

e. Un trato amable y respetuoso de las personas facilitadoras hacia el grupo de participantes durante la intervención recreativa determina, en gran medida, un ambiente agradable que le permite a las personas adultas mayores desenvolverse libremente, disfrutar la actividad e identificarse con el grupo, condición que se presentó en el proceso de investigación.

f. De acuerdo con los resultados de la investigación, la población en estudio presentó mayor concentración y expresó gran satisfacción con aquellas actividades en las que se obtuvo un resultado tangible y se involucró el aprendizaje, como se evidenció en las actividades de cocina, charla motivacional para la siembra del árbol y la construcción del cajón.

g. Las personas participantes en el estudio mostraron, además, un gran deseo por interactuar con la sociedad; el paseo y la noche artística se convirtieron en las actividades más concurridas tanto por quienes participaban en el estudio como por el resto de las personas residentes del Albergue.

La Organización Mundial de la Salud [OMS], (2002) apunta que la falta de apoyo social se asocia con el aumento de la mortalidad, la morbilidad, la angustia psicológica y la disminución de la salud y el bienestar; además considera a la interrupción de los vínculos personales, la soledad y las interacciones conflictivas como fuentes importantes de estrés, mientras que las redes sociales de apoyo son percibidas como fuentes vitales de fortaleza emocional.

h. Es necesario contar con un diagnóstico que vislumbre información detallada 
de las personas participantes en actividades recreativas antes de planificar e implementar los programas. La condición de salud, la posibilidad de movilizarse, el uso de prótesis dentales, sillas de ruedas y andaderas son fundamentales para ofrecer un servicio con la capacidad de adaptarse a las necesidades propias de las personas participantes.

i. Involucrar a la mayor cantidad posible de personas externas a la población del estudio en el proyecto permitió crear sensibilidad social hacia la problemática vivida por las personas adultas mayores. Según Sánchez et ál, (2007) el servicio de voluntariado contribuye a la satisfacción personal de los individuos.

j. El éxito obtenido en la logística, la programación y la implementación de la investigación fue producto de los conocimientos y la experiencia alcanzados en los cursos recibidos durante la Maestría en Recreación.

k. La experiencia adquirida durante la investigación reafirma la capacidad del estudiantado para ejecutar la puesta en práctica de la profesión en el ámbito laboral. El desarrollo de los programas recreativos en el entorno comunitario requieren, además, de una excelente aplicación, una exitosa planificación y mercadeo, hecho que exigen del profesional en Recreación una preparación y una experiencia integral que comprende la investigación, la ejecución de programas recreativos y la propuesta de estos.

1. Este proyecto contribuyó, con el recurso bibliográfico en Recreación, existente en el país, el cual es relativamente escaso y considerado una de las limitaciones más importantes que impiden la fundamentación de ideas y de experiencias en las investigaciones.

\section{Sugerencias con base en esta investigación}

a. Ofrecer una inducción a las personas participantes acerca de los beneficios de la recreación, de tal forma que permita formar en la población participante un criterio positivo acerca del uso adecuado del tiempo libre (Salazar, 2007; Stumbo, 2000; Valdez, Román y Cubillas, 2005).

b. Realizar actividades que les permitan a las personas participantes identificar la distribución de las obligaciones durante el día y complementar con actividades recreativas semanales que ellos mismos se propongan realizar (Stumbo, 2000).

c. En las actividades manuales es preferible que obtengan como resultado un accesorio que satisfaga alguna de sus necesidades o se realice para un fin que le permita a la persona participante sentirse útil. Algunas de las manualidades podrían ser los acomodadores de objetos personales, murales para la sala multiusos, recuerdos para actividades, confección de regalos para población infantil necesitada, entre otros.

d. Promover la participación en actividades cooperativas. González y González (2002) manifiestan que las actividades físicas cooperativas en personas adultas mayores mejoran el bienestar físico, facilitan las relaciones interpersonales y favorecen el desarrollo personal.

e. Tomar en cuenta la necesidad de las personas adultas mayores del Albergue y la interacción con la comunidad. Las visitas de personas al Albergue, los convivios con grupos de otros centros, las salidas a lugares de recreo o a otras instituciones como escuelas o albergues infantiles, entre otros, pueden ser consideradas como opciones recreativas por promover para esta población. De acuerdo con 
Durán, Orbegoz, Uribe-Rodríguez y Uribe (2008), la participación en los sistemas sociales tiende a disminuir en la etapa de adultez mayor, lo cual refuerza la marginación social y ocasiona la pérdida de satisfacción proveniente del entorno comunitario.

\section{Propuesta recreativa}

Los resultados de la investigación reflejaron las condiciones de la población de estudio. Esta información contribuyó a la redacción de una propuesta recreativa que se adecúe a las condiciones propias de las personas adultas mayores del Albergue San José Obrero, del cantón de Siquirres.
La propuesta recreativa pretende que las personas residentes de esta Institución se apropien de una herramienta que les permita conocer los beneficios de aprovechar adecuadamente el tiempo libre con una opción recreativa. Esta propuesta, de igual forma que podría contribuir con la calidad de vida de las personas adultas mayores del hogar, podría servir de modelo a iniciativas recreativas en otras instituciones de atención a la persona adulta mayor.

Las 12 actividades recreativas planteadas en esta propuesta persiguen el objetivo de crear una cultura recreativa en la población, generar situaciones en las que puedan movilizarse, interactuar con la sociedad y sentirse útiles. El programa podría repetirse cada tres meses para facilitar la continuidad del servicio recreativo brindado en el hogar.

Las actividades propuestas son:

Tabla 3

Cronograma de Actividades

\footnotetext{
Propósito

1. Promover en las personas participantes el conocimiento acerca de los beneficios de la recreación y el reconocimiento de su tiempo libre diario.

2. Estimular la coordinación de la motora fina y el sentido de utilidad en las personas participantes.

3. Estimular la coordinación de la motora gruesa y el movimiento de los miembros superiores e inferiores por medio de la realización de movimientos coreográficos de baile.

4. Fomentar en la persona participante el sentido de utilidad y de servicio a la comunidad por medio de la realización de un cuento ilustrado, dirigido a la población infantil.

5. Fomentar en la persona participante el sentido de pertenencia social por medio de la interacción intergeneracional.

6. Estimular la coordinación de la motora fina y el sentido de utilidad en las personas participantes por medio de la realización de una huerta.
} "Conociendo acerca de la
recreación"

"Preparando bizcochos"

\section{"Bailando tropical"}

"Construyo un cuento"

"Nos visitan los niños"

"Cultivo hortalizas"
Persona asesora recreativa

Responsable

Persona asesora recreativa

Persona asesora recreativa

Persona asesora recreativa

Persona asesora recreativa

Persona asesora recreativa 


\begin{tabular}{|c|c|c|}
\hline Propósito & Actividad & Responsable \\
\hline $\begin{array}{l}\text { 7. Estimular en la persona participante la habilidad } \\
\text { mental por medio de la participación en juegos de } \\
\text { mesa. }\end{array}$ & "Rally de juegos de mesa" & $\begin{array}{l}\text { Persona asesora } \\
\text { recreativa }\end{array}$ \\
\hline $\begin{array}{l}\text { 8. Estimular la coordinación de la motora fina, la } \\
\text { creación artística y la imaginación en las personas } \\
\text { participantes por medio de la elaboración de un mural } \\
\text { alusivo a efemérides del mes. }\end{array}$ & "Construyo un mural" & $\begin{array}{l}\text { Persona asesora } \\
\text { recreativa }\end{array}$ \\
\hline $\begin{array}{l}\text { 9. Estimular en la persona participante la habilidad } \\
\text { mental y la interacción social por medio de la partici- } \\
\text { pación en este juego. }\end{array}$ & "El bingo" & $\begin{array}{l}\text { Persona asesora } \\
\text { recreativa }\end{array}$ \\
\hline $\begin{array}{l}\text { 10. Estimular la coordinación de la motora fina, la crea- } \\
\text { tividad y el sentido de utilidad en las personas parti- } \\
\text { cipantes por medio de la confección y la decoración de } \\
\text { un bolso para uso personal. }\end{array}$ & "Construyo un bolso" & $\begin{array}{l}\text { Persona asesora } \\
\text { recreativa }\end{array}$ \\
\hline $\begin{array}{l}\text { 11. Estimular en la persona participante el sentido de } \\
\text { pertenencia a la sociedad por medio de la visita a un } \\
\text { centro recreativo. }\end{array}$ & "El paseo" & $\begin{array}{l}\text { Persona asesora } \\
\text { recreativa }\end{array}$ \\
\hline $\begin{array}{l}\text { 12. Estimular en la persona participante la destreza men- } \\
\text { tal y la interacción grupal por medio de la narración } \\
\text { de historias propias, relacionadas con la música de } \\
\text { épocas de su juventud. }\end{array}$ & "El recuerdo" & $\begin{array}{l}\text { Persona asesora } \\
\text { recreativa }\end{array}$ \\
\hline
\end{tabular}

Fuente: elaboración propia.

La descripción de cada actividad comprende: el nombre asignado, la justificación, el tipo de actividad, el espacio de trabajo, los materiales necesarios, el tiempo asignado, los objetivos a lograr, la intencionalidad, la organización requerida, los pasos a seguir para su desarrollo, las reglas a cumplir, las variantes que se pueden realizar, las sugerencias para ejecutarla y la fuente de donde se obtuvo.

A continuación se muestra un ejemplo de una de las actividades recreativas de la propuesta:

\section{Actividad \#2}

- Nombre: "Preparando bizcochos".

- Justificación: la preparación de bizcochos es una forma de expresar la creatividad y las habilidades de creación; este tipo de actividades en la adultez mayor son de gran importancia debido a que permite aumentar la autoestima, fortalecer la coordinación de la motora fina y servir como medio de interacción social (Garita et al., 2004). En los resultados obtenidos de la investigación realizada por Romero (2010), la sección de cocina resultó muy agradable para las personas participantes.

- Tipo de actividad: pasiva

- Espacio de trabajo: mesa de trabajo/ salón de actividades

- Material: masa, queso maduro rallado, natilla, agua, guantes, gorros para el cabello y jabón antibacterial.

- Tiempo asignado: 1 hora

- Objetivo: Elaborar empanadas de masa con queso para merendar a la hora del café.

- Intencionalidad: que la persona participante ejercite sus manos y sea protagonista en el proceso de la elaboración de sus alimentos. 
- Organización: se requiere de una persona líder que explique la actividad y las reglas, además de que administre el tiempo asignado y distribuya los materiales para cada persona participante.

- Explicación y desarrollo: la actividad consiste en que las personas participantes realicen empanaditas de masa con queso para luego merendar a la hora del café.

\section{- Pasos por seguir:}

1. Desinfectar y limpiar el área de trabajo.

2. A cada persona participante se le dará un gorro para cubrirse la cabeza.

3. Se entregará una porción de jabón y se llevará a las personas a lavarse las manos.

4. A las personas se les pondrán los guantes y se les asignará un lugar en la mesa.

5. Se distribuirán los materiales para iniciar la elaboración de los bizcochos.

6. Se hornearán los bizcochos.

7. Una vez retirado del horno y frío el pan, se procederá a degustar durante el café de la tarde los bizcochos realizados.

8. Al finalizar la actividad se comentará la experiencia vivida.

- Reglas:

1. Respetar a todas las personas participantes y a facilitadores.

2. Respetar el tiempo asignado a la actividad.

3. Respetar el sitio y los materiales asignados a las demás personas.

- Variantes: en lugar de bizcochos, se pueden realizar rosquillas o tortillas de queso.

- Sugerencias: se puede solicitar la colaboración de una persona experta en cocina para que explique la elaboración de los bizcochos.

- Fuente: Bach. Anabelle Vega. Profesora de Educación para el Hogar. Liceo de Pocora.

\section{Para la ejecución de estas actividades} se debe tener en cuenta:

a. La aplicación de la propuesta debe ser dirigida por personas con gran compromiso de servicio y respeto hacia las personas adultas mayores.

b. Las medidas de seguridad durante las actividades deben ser rigurosas; se recomienda asignar a un voluntario por persona, de manera que pueda estar pendiente de si la persona pierde el equilibrio corporal.

c. Realizar talleres de recreación con las personas funcionarias de la Institución, lo que podría contribuir con la sensibilización del personal operativo con respecto a la importancia de la realización de la propuesta recreativa a la población residente del Albergue.

d. La aplicación de esta propuesta en otros centros de atención a la persona adulta mayor requerirá de la adaptación al entorno y las necesidades e intereses recreativos de la población meta. Se recomienda por lo tanto desarrollar un diagnóstico antes de ejecutar las actividades aquí descritas.

\section{Referencias bibliográficas}

Asociación Gerontológica Costarricense [AGECO]. (2008). Ageco. Recuperado el 5 de septiembre del 2008 de http:// www.ageco.org/index.html

Ballestero, C. (1999). Modificaciones del estado físico, anímico, calidad de vida y autoestima en adultos mayores por medio de un programa de actividad sistémica de Karate Do. (Tesis de Maestría sin publicar), Universidad de Costa Rica, San José, Costa Rica.

Butts, D. y Chana, K. (2007, Julio-Agosto). Activities designed to bring the generations together can improve 
well-being and quality of life in older adults. The Journal on Active Aging, 34-39. Recuperado el 5 de septiembre del 2008 de: http://www.icaa.cc/ convention.htm

Cárdenas, M. y Osorio, E. (2006). Iniciativa para la articulación de la recreación para las políticas del envejecimiento. funlibre. Recuperado el 25 de setiembre del 2008 de http://www.funlibre. org/documentos/AdultoMayor.htm

Consejo Nacional de la Persona Adulta Mayor [CONAPAM]. (1999). Ley $N^{\circ}$ 7935. Ley integral para la persona adulta mayor. Recuperado el 15 de noviembre del 2010 de http://www. conapam.go.cr/ley7935.htm

Consejo Nacional de la Persona Adulta Mayor [CONAPAM]. (2008). Consejo Nacional de la Persona Adulta Mayor. San José, Costa Rica. Recuperado el 5 de agosto del 2008 de http://www. conapam.go.cr/index1.asp

Costa Rica. Centro Centroamericano de Población [CCP]. (2009). Indicadores demográficos. San José, Costa Rica. Recuprado el 29 de setiembre del 2009 de http://ccp.ucr.ac.cr/observa/ CRindicadores/inmigra.html

Costa Rica. Instituto de Estadística y Censo [INEC]. (2008). Encuesta de hogares de propósitos múltiples. San José, Costa Rica. Obtenido el 20 de agosto del 2008 de http://www.inec.go.cr/bo tonesAccesoDir/04EncuestaDeHogar es/01Set.html

Costa Rica. Ministerio de Planificación Nacional y Política Económica [MIDEPLAN]. (2008). Población estimada y proyectada. Ministerio de Planificación Nacional y Política Económica, San José, Costa Rica. Recuperado el 20 de agosto del 2008 de http://intranet.mideplan.go.cr/ sides/social/01-03.htm

Durán, D., Orbegoz, L., Uribe-Rodríguez, A. y Uribe, J. (2008,Enero-Abril). Integración social y habilidades funcionales en adultos mayores. Universitas Psicológica, 7(1), 263 270. Recuperado el 26 de setiembre del 2009 de http://scielo.bvspsi.org.br/scielo.php?pid=S1657 $92672008000100019 \&$ script $=$ sci_ arttext

Escobar, J. (2003, Diciembre). Caracterización metodológica para la intervención práctica, en actividad física y salud en adultos mayores. Efdeportes.com, Año 9, $\mathrm{N}^{\circ} 67$. Recuperado el 05 de agosto del 2008 de http://www.efdepoteres.com

Ferreyra, S., Choque, N., y Velazco, V. (2005). La recreación de los ancianos en el Hogar "Padre Lamónaca", Córdoba. Recuperado el 26 de septiembre del 2009 de http://www. cori.unicamp.br/jornadas/completos/ UNC/CA2012Ferreyra-Trab.comp. doc

Figueredo, N., Sotolongo, I., Arcias, R., y Díaz, G. (2003, Setiembre-Diciembre). Caracterización del adulto mayor en la comunidad. Cubana Enfermer, 19(3). Obtenido el 26 de septiembre del 2009, de: http://www.bvs.sld.cu/ revistas/enf/vol19_3_03/enf08303. htm

Garita, J., Godínez, I., López, P., Navarro, R. y Sibaja, R. (2004). Programa recreativo de enfermería para favorecer la construcción de un proyecto de vida de las personas adultas mayores del Centro Gerontológico de Curridabat en el año 2004. (Tesis de Licenciatura sin publicar), Universidad de Costa Rica, S. J., Costa Rica. 
González, L. y González, C. (2002, Setiembre). El voleibol como actividad física recreativa en la tercera edad. Efdeportes.com, Año 8, $\mathrm{N}^{\circ} 52$. Recuperado el 29 de agosto del 2009 de http://www.efdeportes.com/efd52/ recr.htm

Hernández, R., Fernández, C. y Baptista, P. (2006). Metodología de la investigación (3a ed.). México: McGraw-Hill.

Jara, O. (1998). Para sistematizar experiencias. San José, Costa Rica: Editorial Alforja.

Jordan, D., DeGraaf, D. y DeGraaf, K. (2005). Programming for parks, recreation, and leisure services: A servant leadership approach. Pennsylvania: Venture Publishing.

Los Santos, S. (2002, Febrero). La recreación en la tercera edad. Efdeportes. com, Año 8, $\mathrm{N}^{\circ}$ 25. Recuperado el 20 de agosto del 2007 de http://www. efdeportes.com/efd45/tedad.htm

Malmberg, J., Miilumpalo, S., Pasanen, M., Vouri, I. y Oja, P. (2006, Abril). Association of leisure-time physical activity with mobility difficulties among middle-aged and older adults. Journal of Aging and Physical Activity, 14(2), 133-153. Recuperado el 20 de agosto del 2008 de http://www. humankinetics.com/japa/viewarticle. cfm?aid=8387

Marín, M. y García, A. (2004). Calidad de vida en la tercera edad desde la salud y el estado de bienestar psicosocial. Mapfre Medicina, 15(3), 177-185. Obtenido el 20 de agosto del 2008, de: http://dialnet.unirioja.es/servlet/ articulo? codigo $=1064967$

Márquez, J.,y Meléndez,L. (2002). Promoción humana en el envejecimiento una experiencia en la orientación gerontológica. Educere, 6(18), 176 180. Obtenido el 19 de septiembre del 2009, de: http://148.215.1.166:89/ redalyc/pdf/356/35601807.pdf

Mora, M. (2002). Perspectiva subjetiva de la calidad de vida del adulto mayor, diferencias ligadas al género, edad y la práctica de actividad físico recreativa en 21 centros diurnos costarricenses. (Tesis de maestría sin publicar), Universidad Nacional, Heredia, Costa Rica.

Ocampo, R. (2004). Vejez y discapacidad: Visión comparativa de la población adulta mayor rural. (Tesis de Maestría sin publicar), Universidad de Costa Rica, San José, Costa Rica.

Organización Mundial de la Salud [OMS]. (2002). Envejecimiento activo: Un marco político. Revista Especializada de Geriatría y Gerontología, 37(S2): 74-105. Obtenido el 3 de octubre del 2009, de: http://www.imsersomayores.csic.es/documentos/documentos/ oms-envejecimiento-01.pdf

Restrepo,S., Morales, R., Ramírez,M.,López, M. y Varela, L. (2006, Diciembre). Los hábitos alimentarios en el adulto mayor y su relación con los procesos protectores y deteriorantes en la salud. Revista chilena de nutrición, 33(3). Obtenida el 24 de octubre del 2010 de http://www.scielo.cl/scielo. php?script $=$ sci_arttext\&pid $=$ S0717 . $75182006000500006 \& l a n g=p t$

Rico, C. (1999). Recreación y adulto mayor. funlibre. Recuperado el 22 de agosto del 2008, de: http://www.redcreacion. org/articulos/terceraedad.html

Romero, G. (2010). Análisis de necesidades e intereses recreativos de las personas adultas mayores del Albergue San 
José Obrero del cantón de Siquirres. Propuesta recreativa. (Tesis de Maestría sin publicar), Universidad de Costa Rica, San José, Costa Rica.

Salazar, C. G. (2007). Recreación. San José, Costa Rica: Editorial UCR.

Salazar, C. G. (2008). Programación en la recreación. Antología del curso PF-4904 Programación en la Recreación de la Maestría en Recreación. Escuela de Educación Física y Deportes, Facultad de Educación, Universidad de Costa Rica. San José, Costa Rica. (Manuscrito sin publicar).

Sánchez, M., Butt, D., Hatton-Yeo, A., Henkin, N., Jarrott, S., Kaplan, M., Martínez, A., Newman, S., Pinazo, S., Sáez, J. y Weintraub, A., (2007). Programas intergeneracionales. Hacia una sociedad para todas las edades. Barcelona, España: Fundación La Caixa.

Sandín, M. P. (2003). Investigación cualitativa en educación. Fundamentos y tradiciones. Madrid: McGraw Hill.

Sport and Recreation Queensland. (2008). Industry information: Recreation planning. Recuperado el 29 de octubre del 2008 de http://www.sportrec.qld. gov.au/industry_information/recreation_planning/recreation_planning. cfm?\&ITEM

Stumbo, N. (2000). Leisure education I: $A$ manual of activities and resources. Pennsylvania, USA: Venture Publishing.

Tamer, N. (1999). Calidad y equidad en la educación de los adultos mayores. Exigencias y alcances de una demanda pendiente. Escuela Abierta, 3, 103-132. Obtenido el 07 de agosto del 2010, de: http://dialnet.unirioja. es/servlet/dcfichero_articulo?codigo $=$ $195862 \&$ orden $=73670$

Thang, L. (2005). Experiencing leisure in later life: A study of retirees and activity in Singapore. Journal of Cross Cultural Gerontology, 20(4), 307-318.

Valdez, E., Román, R. y Cubillas, M. (2005, Enero-Junio). Análisis de la oferta de servicios para adultos mayores y sus necesidades manifiestas. Psicología y Salud, 15(1), 127-133. Recuperado el 14 de noviembre del 2010 de http://redalyc.uaemex.mx/ pdf/291/29115114.pdf 